\title{
The significance of Brf1 overexpression in human hepatocellular carcinoma
}

\author{
Qian Zhong ${ }^{1, *}$, Shaoyan $\mathbf{X i}^{1, *}$, Jianzhong Liang ${ }^{1, *}$, Ganggang Shi ${ }^{2}$, Yi Huang ${ }^{3}$, Yanmei \\ Zhang ${ }^{2,3}$, Daniel Levy ${ }^{3}$, Shuping Zhong ${ }^{2,3}$ \\ ${ }^{1}$ State Key Laboratory of Oncology in South China, Sun Yat-sen University Cancer Center, Guangzhou, China \\ ${ }^{2}$ Shantou University Medical College, Shantou, Guangdong, China \\ ${ }^{3}$ Department of Biochemistry and Molecular Biology, Keck School of Medicine, University of Southern California, Los Angeles, \\ CA, USA \\ *These authors have contributed equally to this work
}

Correspondence to: Shuping Zhong, e-mail: szhong@usc.edu

Keywords: Brf 1, Pol IIl genes, hepatocellular carcinoma, survival, alcohol

Received: September 08, $2015 \quad$ Accepted: December 07, $2015 \quad$ Published: December 18, 2015

\section{ABSTRACT}

Brf1 (TFIIB-related factor 1) plays a crucial role in cell transformation and tumorigenesis. However, the significance of Brf1 expression in human HCC (hepatocellular carcinoma) cases remains to be addressed. In this study, biopsies of human HCC, liver tumor samples of mice and cell lines of normal and tumor liver were utilized to determine the alteration of Brf1 expression using cytological and molecular biological approaches. Brf1 expression is increased in human HCC cases, which is correlated with shorter survival times. Levels of Brf1 and Pol III (RNA polymerase III-dependent) gene transcription in HCC patients with alcohol consumption are higher than the cases of non-HCC with or without alcohol intake. Induction of Brf1 and Pol III genes by ethanol in hepatoma cells is higher than in non-tumor cells. Ethanol increases the rate of cell transformation. Repression of Brf1 inhibits alcohol-promoted cell transformation. Alcohol consumption enhances Brf1 expression to promote cell transformation. These studies demonstrate that Brf1 is a new biomarker of HCC.

\section{INTRODUCTION}

Alcohol-induced liver injury, including liver steatosis, inflammation, fibrosis and cirrhosis, increases the risk of development of HCC (hepatocellular carcinoma) [1]. Alcohol combines with viruses (hepatitis B or C), carcinogens (aflatoxin), obesity, or diabetes mellitus to promote liver cancer development [2-5]. However, the mechanisms of alcohol-induced HCC and the significance of Brf1 (TFIIB-related factor 1) expression in HCC remain to be elucidated. Previous studies have shown that chronic alcohol consumption results in the production of acetaldehyde. Acetaldehyde is a by-product of alcohol metabolism catalyzed by ADH (alcohol dehydrogenase), which has direct mutagenic and carcinogenic effects in vitro and in vivo [6-8]. Although the exact mechanism by which alcohol causes HCC is still unclear, alcohol consumption is thought to induce liver carcinogenesis through various mechanisms: mutagenesis by the ethanol metabolite acetaldehyde, and oxidative damage, as well as by affecting the one-carbon metabolic pathways through reduced folic acid intake and use. However, little is known about the role of alcohol in Brfl and Pol III gene (RNA polymerase III-dependent gene) transcription, which is responsible for protein synthesis and tightly linked to cell transformation and tumor development.

RNA Pol III is responsible for the synthesis of a variety of small untranslated RNAs, including $5 \mathrm{~S}$ rRNA and tRNAs, which are elevated in both transformed and tumor cells suggesting that it plays a crucial role in tumorigenesis [9-17]. Brf1 is a key transcription factor, which specifically regulates Pol III gene transcription. 
Our studies have demonstrated that alcohol induces Brf1 expression and Pol III gene transcription in both in vivo and in vitro [11]. Liver tumor development was induced in alcohol-fed NS5A (HCV non-structural $5 \mathrm{~A}$ protein) transgenic mice [12]. Ethanol increased the expression of TFIIIB subunits, TBP, Brf1 and Bdp1 in HepG2-ADH cells [11]. Regulation of Bdp1, but not Brf1, occurred through alterations in TBP expression [13]. A repression in Brf1 expression decreased Pol III gene transcription and was sufficient to inhibit cell transformation [14-17]. Brf1 induction caused an increase in cell proliferation and oncogenic transformation [14-17]. However, it is not clear what role of Brf1 overexpression plays in human cancers. Therefore, in the present studies, we have determined the alteration of Brfl expression and analyzed the significance of its change in human HCC cases. The results indicate that $\mathrm{Brfl}$ is overexpressed in $\mathrm{HCC}$ cases, which results in a shorter overall survival period. Brf1 expression and Pol III gene transcription in HCC cases with alcohol consumption are further increased. Ethanol elevates rate of cell transformation, whereas repression of Brf1 reduces the ethanol-increased the rate of cell transformation. These studies demonstrate that Brf1 is a novel biomarker of HCC, which play critically important role in HCC development.

\section{RESULTS}

\section{The expression of Brf1 in the biopsies of $\mathrm{HCC}$ patients}

Our studies have demonstrated that alcohol induced Brf1 expression and Pol III gene transcription in vitro and in vivo by using cell culture models and NS5A transgenic mice [11, 14-17]. To investigate the significance of Brfl expression in HCC patients, we have carried out immunohistochemistry (IHC) analysis using a specific antibody against Brf1 in tissue microarrays of 133 human HCC tissue samples. The clinical pathological characteristics of $\mathrm{HCC}$ cases were summarized in (Supplementary Table S1). The representative pictures of IHC are shown in Figure 1. Strong signals of Brf1 staining are observed in HCC tissue, compared to para HCC tissue (Figure 1A and 1B). The histological study indicates the appearance of Brfl in the tissues of HCC and para HCC (Figure 1A1, 3). The result reveals that the localization of Brf1 is primarily in the nucleus and cytoplasm in HCC cells with only weak signal of Brfl detected in para HCC tissue (Figure 1A2, 4). We observed staining of BRF1 in all of $133 \mathrm{HCC}$ cases. All the samples were separated into either low or high BRF1 expression groups (Figure 1B). High expression of BRF1 was observed in 98/133 (73.5\%) of HCC samples. The IRS (immunoreactivity score) was multiplied by the score of tumor cell percentage and the score of staining intensity.
Brf1 expression was significantly associated with serum AFP (alpha-fetoprotein) values $(\mathrm{P}=0.028$, Supplementary Table S2) in all HCC patients. However, there was no significant correlation between Brf1 expression and other clinic pathological features, such as patient age, gender, TNM stages, recurrence $(\mathrm{P}>0.05$, Supplementary Table S2). Univariate Cox proportional hazard regression analysis showed that Brfl expression $(\mathrm{P}<0.001)$, tumor diameter $(\mathrm{P}=0.007)$, recurrence $(\mathrm{P}=0.003)$, serum AFP $(\mathrm{P}=0.011)$ and $T N M$ stages $(\mathrm{P}<0.001)$ were significantly associated with overall survival (Table 1). To determine whether BRF1 expression was correlated with OS (overall survival) of HCC patients, we evaluated the prognostic value of Brfl through estimation of OS using Kaplan-Meier and log-rank test analyses. As shown in Figure 2, high Brf1 expression was significantly related to poor OS compared to low Brfl expression (43.43 versus 53.84, $\mathrm{P}=0.044)$. We analyzed the RNA-seq and tumor progression data from "Liver Hepatocellular Carcinoma (TCGA, provisional)" at cBioPortal [18-19] and found that high expression of BRF1 predicts a poor overall survival (20.6 versus 53.3 months, $\mathrm{P}=0.032$, Supplementary Figure S1).

\section{Alcohol-increased Brf1 expression promotes liver tumor development}

The above studies have demonstrated that high expression of Brfl in HCC tissues is correlated to the short overall survival times of the human HCC cases. To investigate the role of alcohol-induced deregulation of Brfl and Pol III genes in HCC, we further explore the relationship of alcohol consumption with Brfl expression. The cellular levels of Brfl protein and mRNA were determined in human normal livers (Nor L), alcohol consumption liver (Acl-L) and alcohol consumptionHCC liver (Alc/HCC-L). The results indicate that protein and mRNA levels of Brfl are increased in Alc/HCC-L, compared to the levels observed in Nor-L and Acl-L (Figure $3 \mathrm{~A}-3 \mathrm{~B})$. In addition, the amounts of precursor tRNA ${ }^{\mathrm{Leu}}$ and 5S rRNA transcript of these samples were measured by RTqPCR. The results reveal that the levels of Pol III genes, both pre-tRNA ${ }^{\text {Leu }}$ (Figure 3C) and 5S rRNA (Figure 3D) are increased in Acl/HCC-L, compared to the transcription levels of these genes in the samples of Nor-L and Acl-L.

To further explore the mechanism of alcoholpromoted liver tumor formation, we utilized the liver tissues (kindly provided by Dr. Machida K) of hepatitis $\mathrm{C}$ virus (HCV) non-structure 5A (NS5A) transgenic mouse to determine the role of Brf1 in this process. The results indicate that the precursor tRNA ${ }^{\text {Leu }}$ and $5 \mathrm{~S}$ rRNA transcript levels are elevated in HCC tissues of alcohol-fed NS5A mice, compared to the levels of the Pol III genes in the liver tissues from non-alcohol-fed and alcohol-fed NS5A mice (Figure 4B-4C). More interestingly, the cellular level of Brfl mRNA in HCC 


\section{A}

A1 Brf1 in HCC

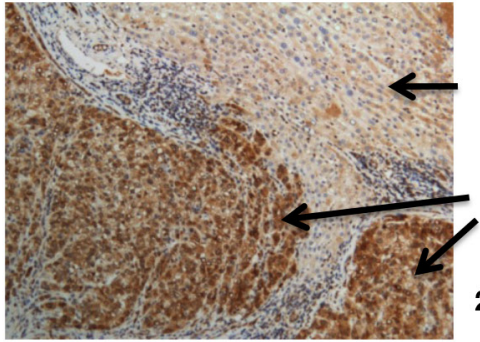

A3

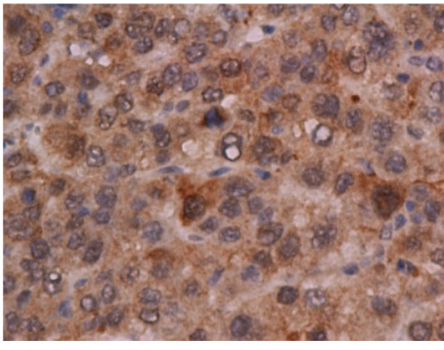

A2 H\&E staining in HCC

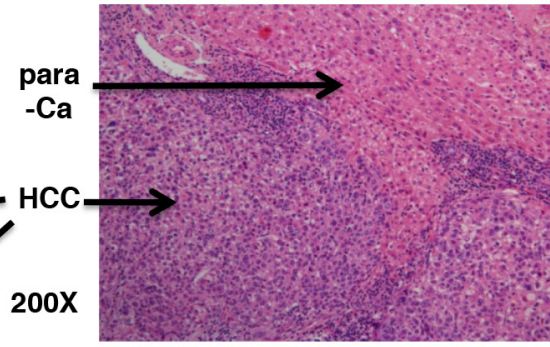

A4 Brf1 in HCC para-Ca

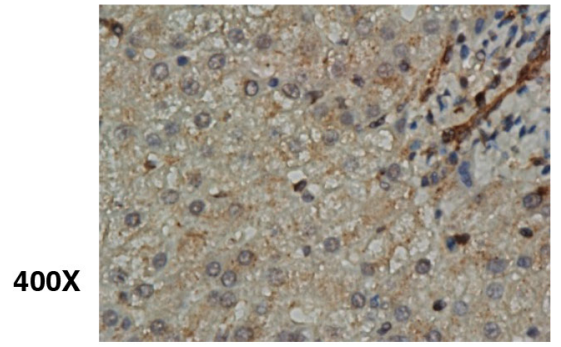

B

B1 Low level of Brf1 in HCC

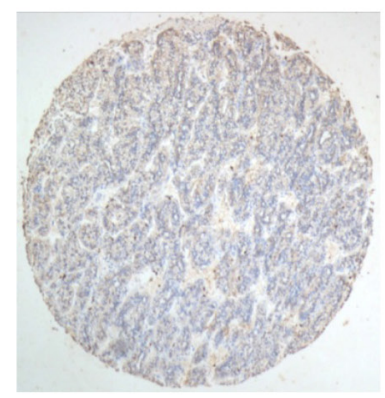

B3

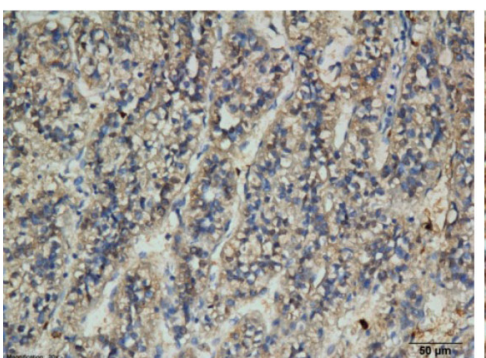

B2 High level of Brf1 in HCC

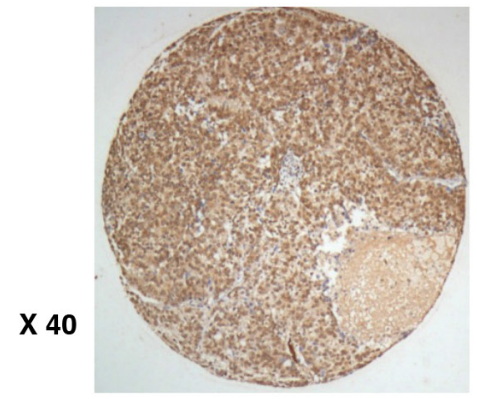

B4

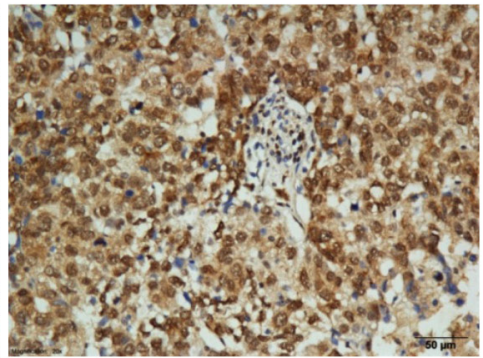

X 200

Figure 1: Immunohistochemistry (IHC) staining of Brf1 in human HCC. A. Brf1 staining. (A1) IHC staining of Brf1 in HCC; (A2) H\&E staining of HCC liver tissue; (A3) IHC staining of Brfl in cancer foci of HCC; (A4) IHC staining of Brfl in the para-Ca tissue of HCC. (A1 \& A2) 200 X magnification; (A3 \& A4) 400 X magnification. A representative staining of Brf1 in HCC. B. Weak and strong staining of Brf1 (B1 \& B3) are low expression of Brf1 in HCC; (B2 \& B4) are high expression of Brf1. (B1 \& B2) 40 x magnification; (B3 \& B4) $200 \mathrm{x}$ magnification.

tissues of alcohol-fed NS5A mice is also significantly higher than in the non-tumor liver tissues of these mice with or without alcohol intake (Figure 4A). These studies using human biopsy samples and mouse liver tissues demonstrate that increases in Brfl and Pol III gene expression are tightly linked to alcohol-associated HCC. 
Table 1: Association of various factors with overall survival in $133 \mathrm{HCCs}$ determined by COX regression model.

\begin{tabular}{|c|c|c|c|c|}
\hline Univariate analysis & Multivariate analysis & & & \\
\hline Variable & $\operatorname{HR}_{a}\left(95 \% \mathrm{CI}_{b}\right)$ & $P_{c}$ & HR(95\%CI) & $P$ \\
\hline $\begin{array}{l}\text { Gender Male vs. } \\
\text { Female }\end{array}$ & $0.728(0.406-1.305)$ & 0.286 & & \\
\hline Age $\leq 50 \mathrm{yr}$ vs. $>50 \mathrm{yr}$ & $1.278(0.803-2.033)$ & 0.301 & & \\
\hline $\begin{array}{l}\text { Tumor diameter } \leq 5 \mathrm{~cm} \\
\text { vs. }>5 \mathrm{~cm}\end{array}$ & $2.060(1.217-3.486)$ & 0.007 & $1.364(0.779-2.386)$ & 0.277 \\
\hline $\begin{array}{l}\text { Serum AFP }(\mathrm{ng} / \mathrm{ml}) \\
<400 \text { vs }>400\end{array}$ & $1.810(1.142-2.867)$ & 0.011 & & \\
\hline Serum ALT $<40$ vs $>40$ & $1.123(0.767-1.921)$ & 0.409 & & \\
\hline $\begin{array}{l}\text { Recurrence Absent vs. } \\
\text { Present }\end{array}$ & $2.281(1.730-4.598)$ & 0.003 & $1.742(1.010-3.005)$ & 0.046 \\
\hline $\begin{array}{l}\text { TNM stage Early vs. } \\
\text { Advanced }\end{array}$ & $4.032(2.512-6.472)$ & 0.000 & $2.760(1.595-4.775)$ & 0.000 \\
\hline $\begin{array}{l}\text { BRF1 expression Low } \\
\text { vs. High }\end{array}$ & $1.823(1.018-3.267)$ & 0.044 & $1.470(0.812-2.663)$ & 0.204 \\
\hline
\end{tabular}

\section{Survival Functions}

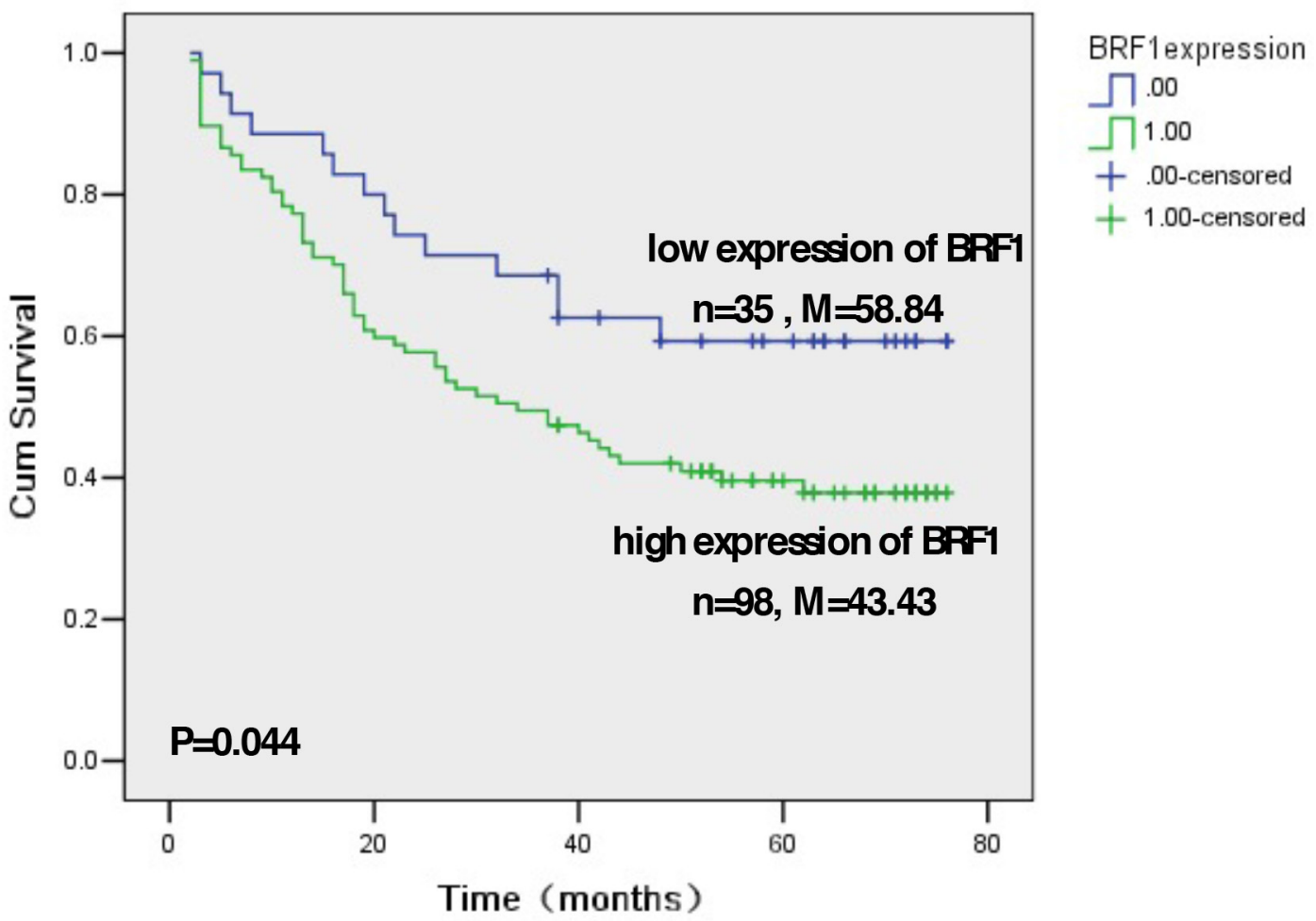

Figure 2: Kaplan-Meier survival curve and log-rank test analysis of the association between Brf1 expression and HCC patient survival. A. Brfl expression of $133 \mathrm{HCC}$ cases wasdetermined by pathological analysis and immunohistochemistry staining. $\mathrm{n}=$ number of patients in the subgroup, $\mathrm{M}=$ median survival in months of the subgroup. 


\section{Difference of Brf1 expression in tumor and non-tumor liver cell lines and the role of Brf1 in alcohol-promoted cell transformation}

The studies from our laboratory and others have demonstrated that reduction of Brfl expression decreases Pol III gene transcription [11, 14-17]. The above studies have demonstrated that Brfl expression is increased in human HCC tissues, compared to the para tissues of HCC. Therefore, we further determined the cellular levels of Brfl in tumor and non-tumor liver cell lines in these systems. The results reveal that the levels of Brfl mRNA and protein in tumor cell lines [TSCML (tumor stem cells of mouse liver) and HepG2-ADH], are higher than the non-tumor line (AML-12), an immortalized liver cell line of mouse (Figure 5A and 5B). Furthermore, the induction of Brf1 expression caused by ethanol in tumor lines is significantly higher than in non-tumor line (Figure 5A and 5B). This suggests that oncogenic cell lines are more sensitive to the alcohol-induced response. In addition, we also found similar results for Pol III gene transcription in these cell lines (Figure 5C and 5D). These results demonstrate that alcohol-induced Brfl and Pol III gene transcription in tumor lines is significantly higher than in non-tumor line. This suggests that higher levels of Brfl are associated with the oncogenic status of these cells. Thus, we further
A

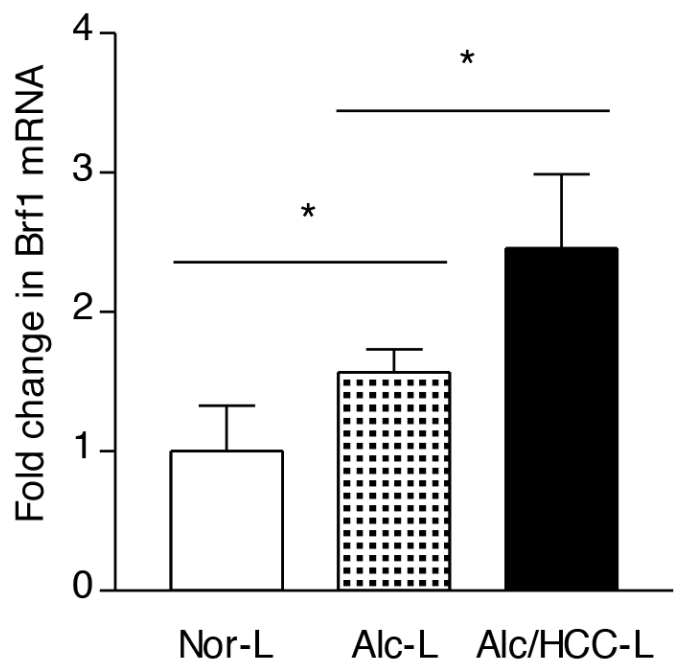

B
C

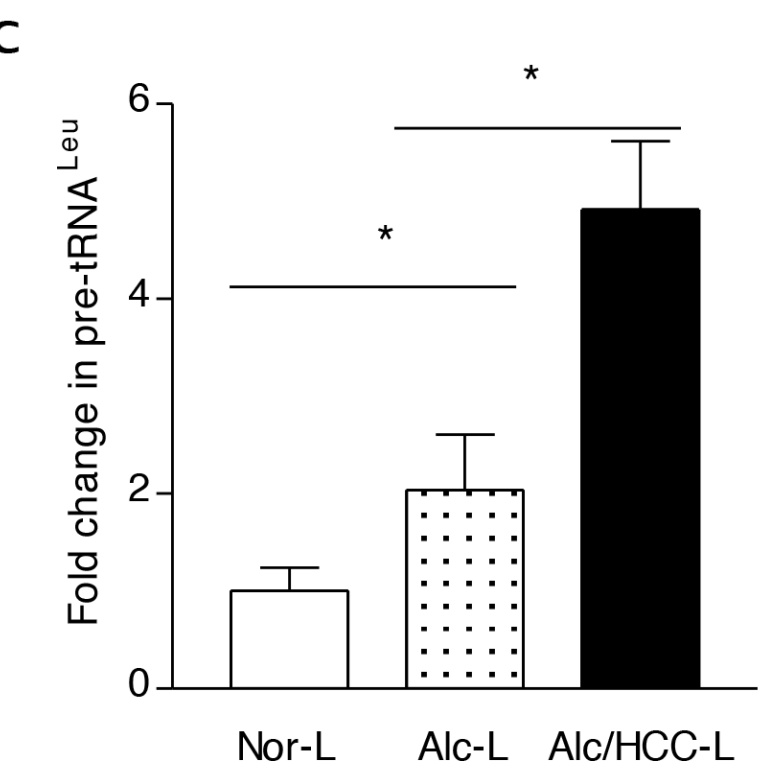

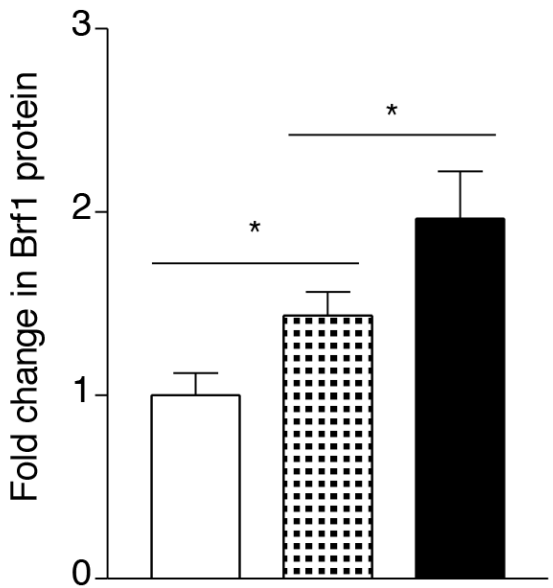

Nor-L Acl-L Acl/HCC-L

0
Alc/HCC-L Alc-L

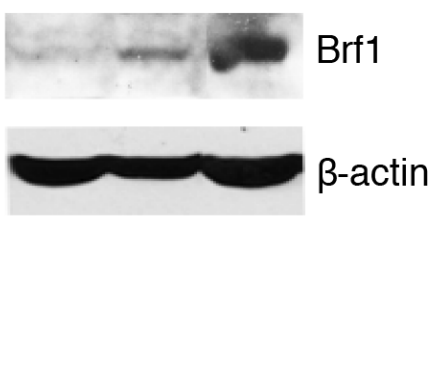

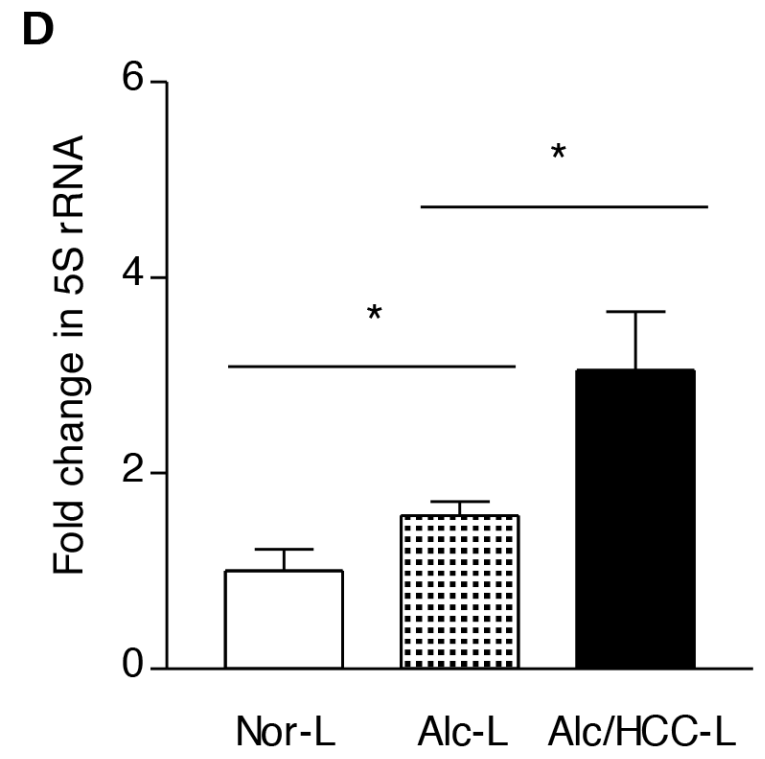

Figure 3: Expression of Brf1 and Pol III genes in HCC cases with alcohol consumption. The total RNA and tissue lysates were extracted from the human liver tissues of normal (Nor-L, $n=6$ ), alcohol consumption (Alc-L, $n=8$ ) and HCC cases with alcohol consumption (Alc/HCC-L, n=7). Immunoblot analysis was performed to determine cellular levels of Brf1 protein (right) and its quantify data (left) B. RT-qPCR was carried out to measure the amounts of Brf1 mRNA A. pre-tRNA ${ }^{\text {Leu }}$ C. 5S rRNA D. The transcription levels of the genes were calculated by normalizing to the amount of GAPDH mRNA. The fold change was normalized by Nor-L. The bars represent Mean \pm SE of at least three independent determinations. ${ }^{*}: \mathrm{p}<0.05$ as indicated. 
determined whether repressing Brfl affects the alcoholinduced phenotype. Our earlier studies have demonstrated that repression of Brfl expression and Pol III gene transcription by Brfl siRNA inhibits cell transformation [15-17, 20]. Here, we established whether alcohol affects the rate of cell transformation and repression of Brfl inhibits alcohol-promoted transformation of AML-12 cells. Our studies have demonstrated that Brfl siRNA efficiently decreases its cellular levels of protein and mRNA $[15-17,20]$. Here, our results show that reduction of Brfl by its siRNA inhibits precursor tRNA ${ }^{\text {Leu }}$ and $5 \mathrm{~S}$ rRNA transcription in TSCML and AML-12 (Figure 6A6D). To further assess the effect of Brfl on alcohol-caused phenotypic alteration, we performed a soft agar assay. The results show that alcohol increases the rate of EGF-caused colony formation of AML-12 cells (Figure 7A), whereas Brfl siRNA represses cell anchorage-independent growth (Figure 7A-7B). This indicates that the change of Brfl levels in the cells results in alteration of alcohol-promoted phenotypes of non-tumor liver cells.

A
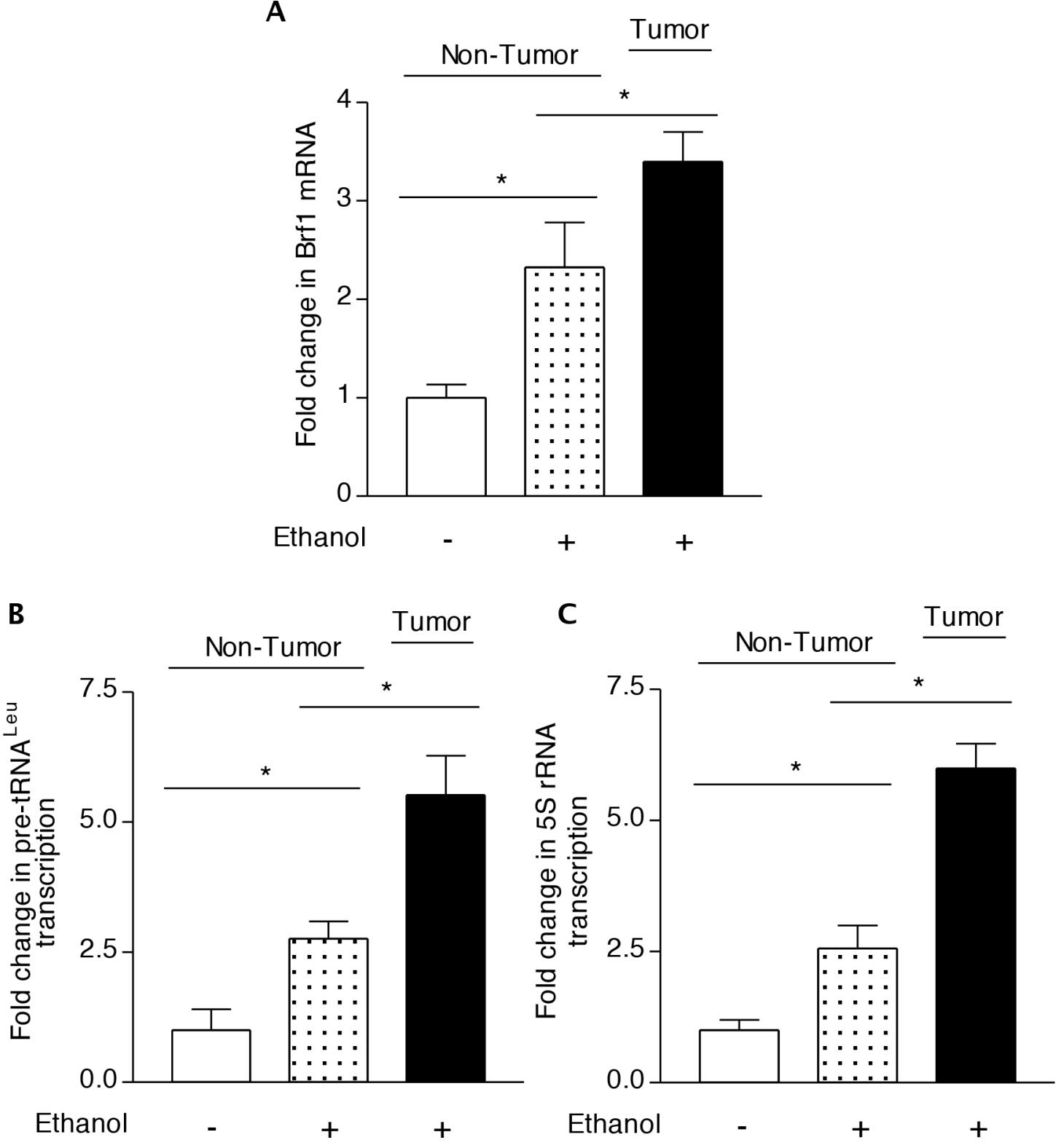

Figure 4: Transcription of Brf1 and Pol III genes in chronic alcohol administration in mice. C57BL/6 transgenic mice harboring the HCV NS5A gene were fed with 3.5\% ethanol in the Lieber-DeCarli liquid diet or control diet for 12 months. Liver tissues were harvested from these mice. RNA was extracted from the non-tumor or tumor portion of the livers and RT-qPCR was used to measure the amounts of Brfl A. pre-tRNA ${ }^{\mathrm{Leu}}$ B. 5S rRNA C. relative to GAPDH. The values represent means $\pm \mathrm{SE}$ from three independent experiments. Each group of mice includes at least five mice. The fold change was calculated in each group by normalizing to the mice fed with control diet. *: $\mathrm{p}<0.05$ as indicated. 


\section{DISCUSSION}

Our results have indicated that Brf1 expression is increased in both human female and male cases of HCC. Further analysis reveals that human HCC cases with high expression of Brf1 have a shorter overall survival period. The cellular level of Brfl in human HCC cases with alcohol consumption is higher than non-HCC cases with or without alcohol intake. Alcohol feeding promotes liver tumor development of NS5A mice, while Brfl expression and Pol III gene transcription are increased in alcohol-fed NS5A mice. Furthermore, our studies demonstrate that the amounts of Brfl and Pol III gene expression in tumor liver cell lines is significantly higher than non-tumor liver cell line. Repression of Brf1 expression reduces alcoholincreased rate of cell transformation. Together, these studies demonstrate that Brfl is a novel biomarker of
HCC, which plays a key role in alcohol-associated liver tumor development.

Studies have indicated that females are more resistant to several diseases, such as HCC [21-22]. HCC is the fifth common cancer and ranks third in annual mortality worldwide [23]. Women have a significantly lower incidence of HCC than men [23]. Diethylnitrosamine (DEN) administration caused HCC in $100 \%$ of male mice [24], but only in $10 \%$ of female mice [25]. Here, our results reveal this feature: $84 \%$ cases $(111 / 133)$ of HCC are the males, but only $16 \%$ cases $(21 / 133)$ are the females, which further supports the idea that females are resistant to $\mathrm{HCC}$ development. Because high expression of Brf1 is associated with shorter overall survival period (Figure 2 and Supplementary Figure S1), this implies that the human HCC cases with high expression of Brfl have a poorer prognosis. Studies have indicated that Foxa1/2
A

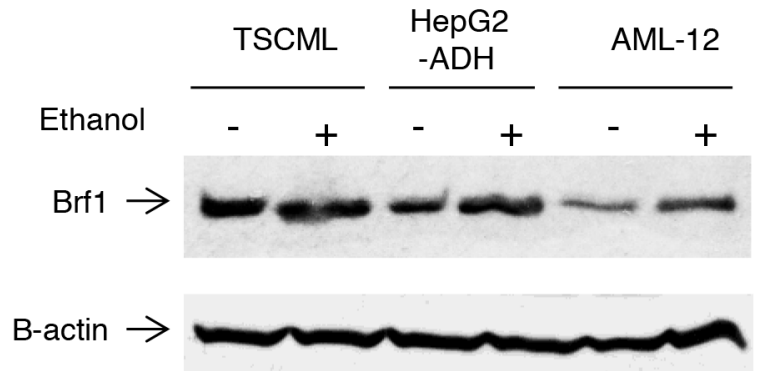

C

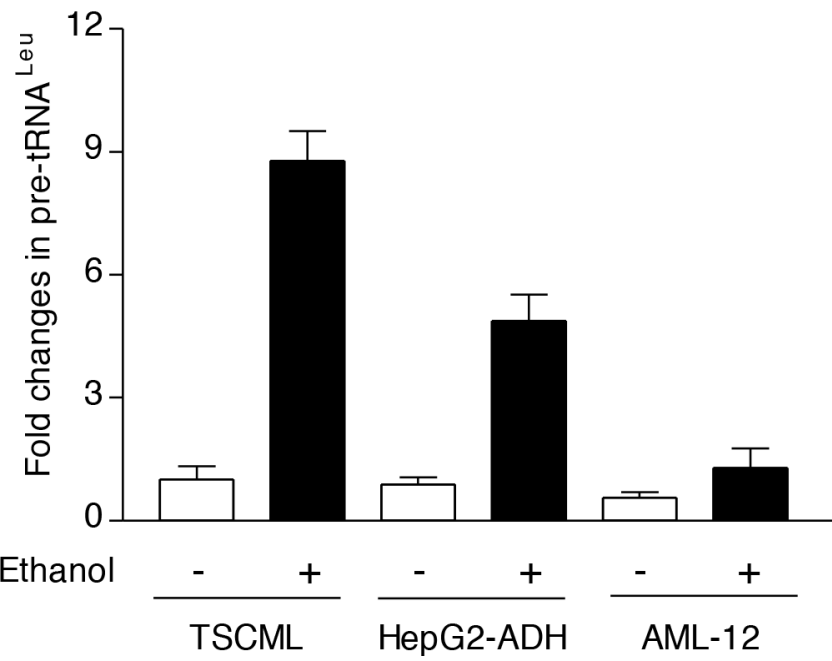

B
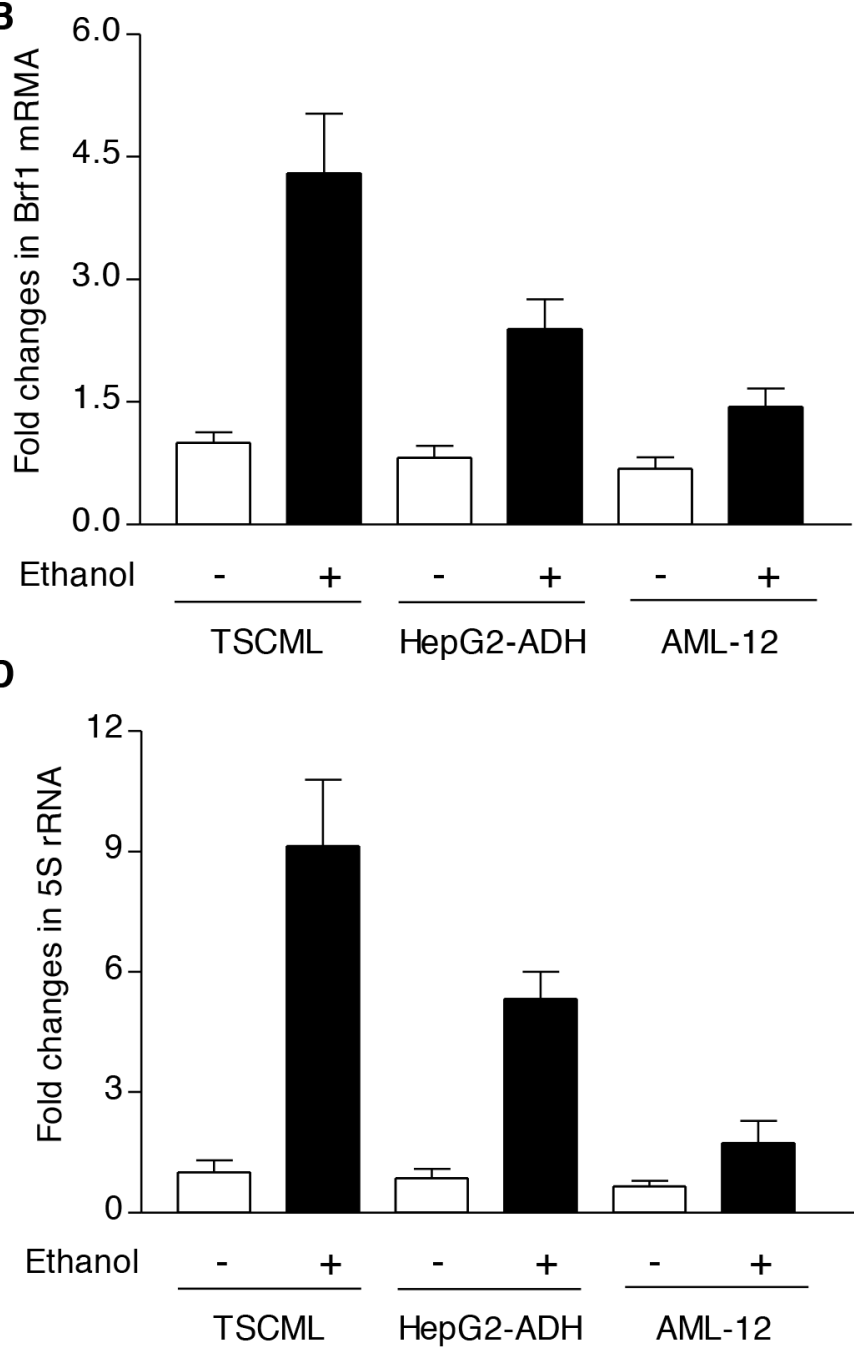

Figure 5: Brf1 and Pol III gene expression in non-tumor and tumor liver cell lines. The cells of TSCML, HepG2-ADH and AML-12 cell lines were starved in DMEM-F12 for 4h. Cells were treated with or without $50 \mathrm{mM}$ ethanol for another $1 \mathrm{~h}$ : The cell lysates were extracted from these cells to determine the levels of Brfl protein by immunoblot analysis A. Total RNAs were extracted from these cells and RT-qPCR was performed to measure the amounts of Brf1 mRNA B. pre-tRNA ${ }^{\text {Leu }} \mathbf{C}$. and 5S rRNA D. The fold change was calculated by normalizing to the amount of GAPDH mRNA. The bars represent Mean $\pm \mathrm{SE}$ of at least three independent determinations. 
A

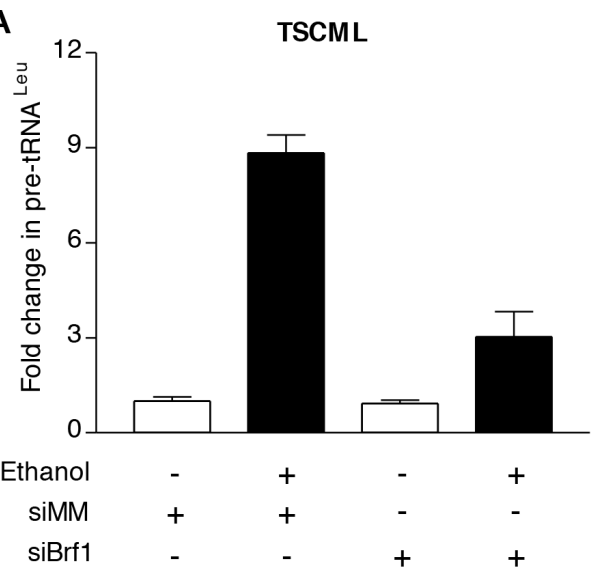

C

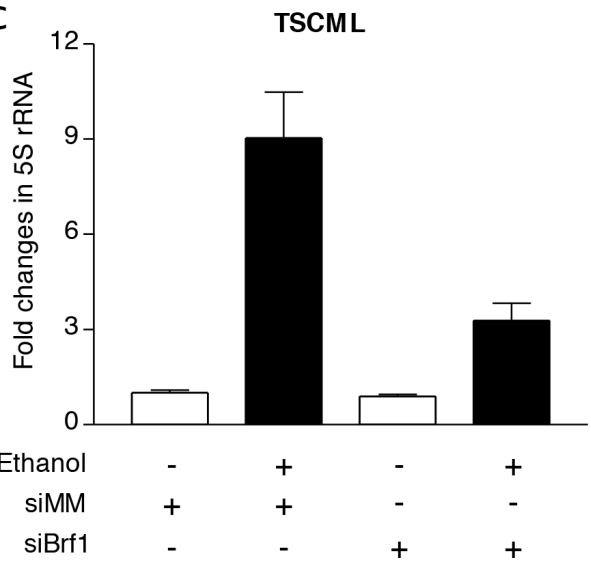

B $12 \quad$ AML-12

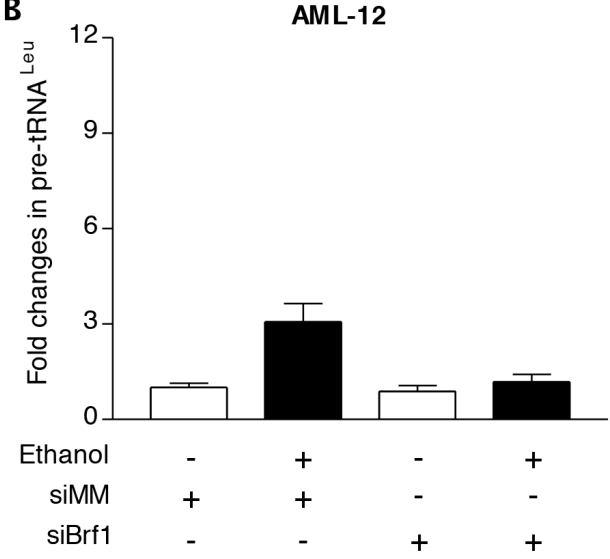

D

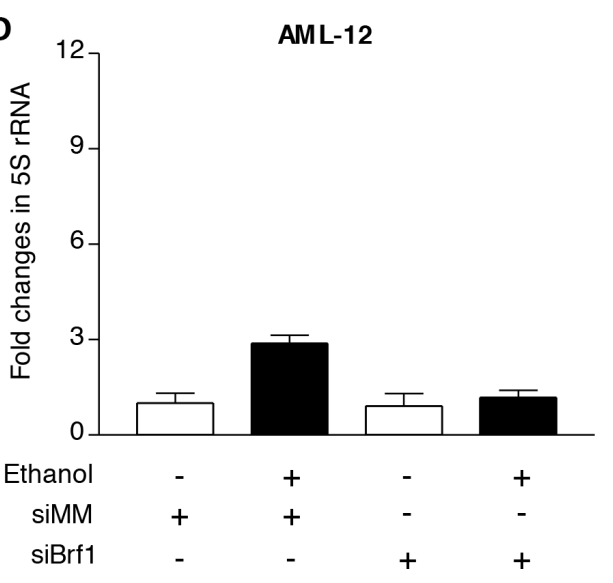

Figure 6: Repression of Brf1 decreases the induction of Pol III genes caused by alcohol. The cells of TSCML and AML-12 were transfected with mismatch RNA (mmRNA) or Brfl siRNA for $48 \mathrm{~h}$. The cells were treated as described in Figure 5. Total RNAs were extracted from these cells and RT-qPCR was performed to measure the amounts of pre-tRNA ${ }^{\text {Leu }} \mathbf{A}, \mathbf{B}$. and 5S rRNA $\mathbf{C}, \mathbf{D}$. The fold change was calculated by normalizing to the amount of GAPDH mRNA. The bars represent Mean \pm SE of at least three independent determinations.

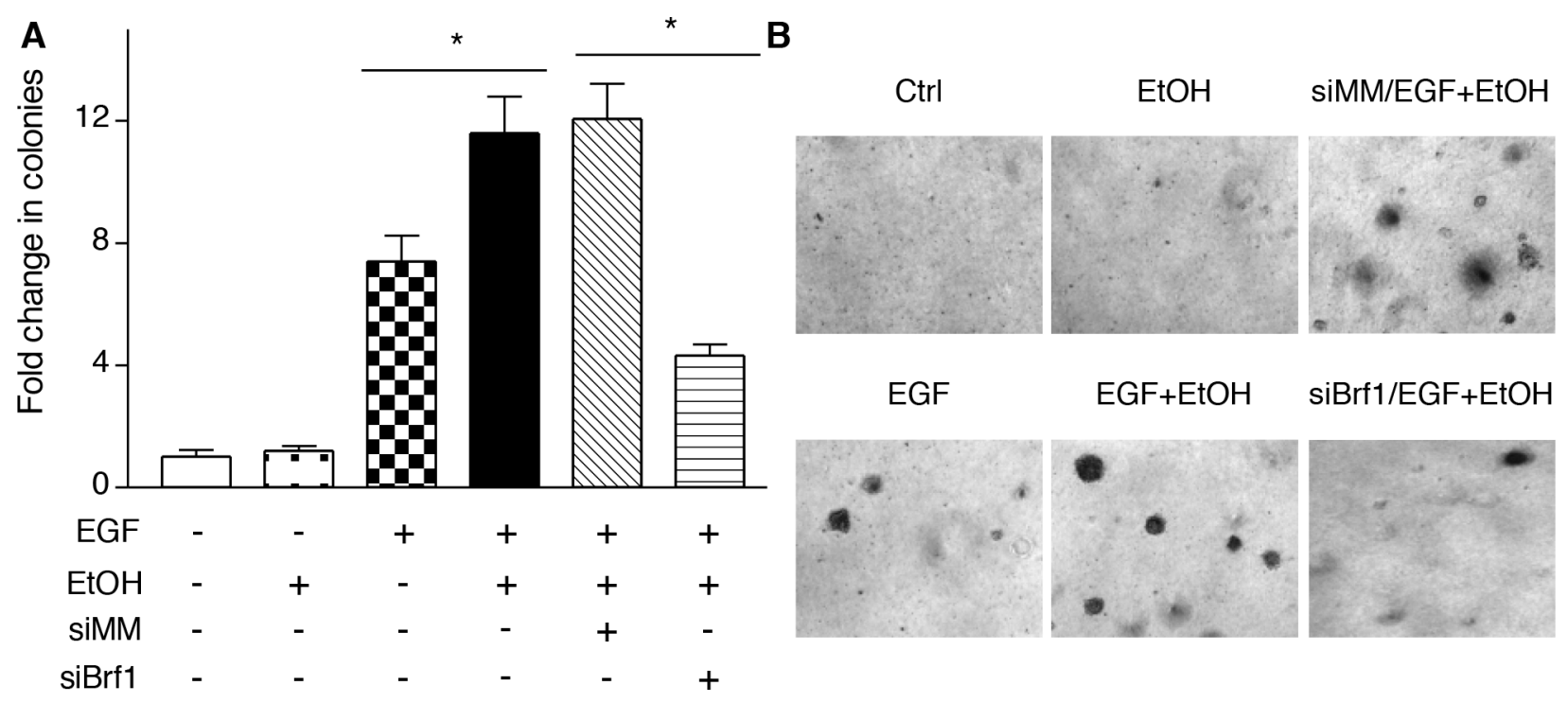

Figure 7: Reducing the amounts of cellular Brf1 inhibits alcohol-increased the rate of cell transformation. AML-12 cells were poured in triplicate into 6-well plate with $0.35 \%$ agar containing $50 \mathrm{mM}$ ethanol or PBS as control and grown in the medium with or without $50 \mathrm{mM}$ ethanol and plus EGF (20ng/ml) for 4 weeks or longer. The cells were analyzed for colony formation in soft agar. Colonies were counted at 3-4 weeks after plating. Values are the means $\pm \mathrm{SE}(\mathrm{n} \geq \mathrm{C} 3) .{ }^{*}: \mathrm{p}<0.05$ as indicated. 
deficient female mice are more susceptible to DENinduced liver cancer development because of the loss of the interaction between Foxa1/2 and ER $\alpha$ [26]. A reason for the susceptibility for HCC development in female and male mice may depend on the interaction of sex hormones with factors, such as Foxa1/2, ER $\alpha$ or AR (androgen). The studies from our laboratory have demonstrated that DEN increases Brfl expression and Pol III transcription in liver cells [16], whereas ER $\alpha$ upregulates alcohol-induced Brf1 expression in breast cells [15]. This suggests that a potential interaction of Foxa1/2, ER $\alpha$ and Brfl may exist, which may modulate Pol III gene transcription. Given that high expression of Brf1 is associated with short overall survival times (Figure 2 and Supplementary Figure S1), reducing the cellular level of Brfl of HCC patients may be used to extend overall survival period and measuring the level of Brfl expression may be used to monitor the efficacy of treatment of HCC.

Oncogenic proteins, such as Ras, c-Jun, and c-Myc, stimulate Pol III gene transcription [10-11, 27-28], whereas tumor suppressors, such as pRb, p53, BRCA1 and PTEN repress transcription of this class of genes [10-11, 27-31]. Studies have indicated that RNA Pol III transcription products are elevated in both transformed and tumor cells suggesting that they play a crucial role in tumorigenesis $[11,14-17,20]$. Consistent with this idea, enhanced Pol III gene transcription is required for oncogenic transformation [13-17, 20]. The ability of these oncogenic and tumor suppressor proteins to regulate Pol III gene transcription result from their capacity to modulate the TFIIIB complex. The TFIIIB complex consists of TATA box-binding protein (TBP) and its associated factors, Brfl and Bdp1. We have demonstrated that enhancement of Brf1 expression and Pol III gene transcription is correlated with liver tumor formation [11]. In contrast, repression of Brfl expression inhibits cell transformation [14-16, 20]. Our studies indicate that alcohol increases expression of Brf1 and Pol III genes [11, 14, 20]. We have demonstrated that alcohol increases cellular level of c-Jun, which occupies to Brfl promoter to enhance Brf1 expression and Pol III gene transcription [11]. Here, the results further indicate that the cases of human $\mathrm{HCC}$ with alcohol consumption reveal higher expression of Brfl and Pol III genes, compared to other groups (Figure 3). The animal studies further suggest that alcohol feeding of mice promotes liver tumor formation (Figure 4). This shows that alcohol-increased expression of these genes is critically important during HCC development. Alcohol has been classified as carcinogenic in human [32-33]. Target sites for alcohol-related carcinogenesis in human include the breast, liver and multiple additional organs. Is there a common mechanism, which enhances alcohol-associated cancer development in different human organs? Cancer cells have a consistent cytological feature of nucleolar hypertrophy, where RNA Pol III genes are transcribed [10]. This feature provides the possibility of elucidating a common mechanism of alcohol-associated human cancers by determining deregulation of Brf1 and Pol III genes. Animal experiments have shown that alcohol intake promotes tumor development $[34,35]$. These studies are consistent with our findings in Figure 4. Furthermore, we have found that alcohol increases expression of Brfl and Pol III genes in liver and breast cell lines and the cellular levels of Brf1 in cancer cell lines are higher than in nontumor cell lines [11, 15-18, 28]. In contrast, repression of Brfl expression inhibits cell transformation [11, 15-17, $20,28]$. The present studies indicate higher level of Brf1 in human tumor tissue of HCC. This suggests that the high level of Brf1 reflects the oncogenesis status of cells and Brf1 may be a novel biomarker for cancers, whereas alcohol-caused deregulation of Brfl and Pol III genes may be a common mechanism of alcohol-associated cancers.

In summary, the present studies provide evidence that Brf1 is overexpressed in human HCC cases, where high expression of Brfl results in shorter overall survival times. Alcohol intake increases cellular levels of Brf1 and Pol III genes expression to promote liver tumor development. Liver tumor cell lines show high levels of Brf1 protein and mRNA, compared to non-tumor cell lines. In contrast, repression of Brfl decreases the induction of Pol III gene transcription caused by alcohol and reduces the rate of alcohol-induced cell transformation. These studies indicate that Brfl could be a biomarker of diagnosis and prognosis of HCC. These findings suggest the possibility that detecting cellular level of Brfl expression may be a potential approach to measure the efficacy of HCC treatment.

\section{MATERIALS AND METHODS}

\section{Hepatocellular carcinoma patients and clinical tissue specimens}

All 133 paraffin-embedded HCC specimens collected for immunohistochemistry (IHC) assay were clinically and histologically diagnosed at Sun Yat-sen University Cancer Center (SYSUCC), Guangzhou, China between 2005 and 2007. Tissue microarrays were constructed from each resected specimen. If the HCC is multiple, then the biggest tumor was selected in this tissue microarray. The clinicopathological characteristics are summarized in Supplementary Table S1. All the samples were obtained during the surgery prior to radiotherapy or chemotherapy. Cancer TNM stage was defined on the basis of the AJCC (American Joint Committee on Cancer). All the patients were followed from the date of diagnosis until death or the latest census date. The informed consent was obtained from each patient and the study was approved from the Institute Research Ethics Committee of Sun YatSen University. 


\section{Reagents and antibodies}

Cell culture medium (DMEM/F12), OPTI-MEM, Lipofectamine 2000 and TRIzol reagent were from Life Technologies (San Diego, CA, USA). Antibody against $\beta$-actin was obtained from Santa Cruz Biotech (Santa Cruz, CA, USA). Mismatch RNA (mmRNA) was described previously [16]. Brf1 antibody was from Bethyl laboratories Inc (Montgomery, TX, USA). The sequences of primers and Brfl siRNA were described in (Supplementary Table S3) [20]. Tumor stem cells of mouse liver (TSCML) were kindly provided by Dr. K. Machida at USC (University of Southern California). AML-12 cell line was from ATCC. HepG2-ADH cell line was kindly provided by Dr. D.L. Clemens (University of Nebraska). The animal experiments were conducted in accordance with the approved institutional animal care and use committee protocol at USC.

\section{Immunohistochemistry staining}

Formalin-fixed, paraffin-embedded HCC tissue microarrays were cut into $4-\mu \mathrm{m}$ thick sequential sections and baked at $65^{\circ} \mathrm{C}$ for 30 minutes. Deparaffinization of sections was performed with xylene two times and at different concentrations of alcohol and rehydrated. These sections were immersed in 3\% hydrogen peroxide for 10 min to block endogenous peroxidase activity at room temperature, and then boiled in Citrate Antigen Retrieval Solution $(\mathrm{PH}=6.0)$ for $5 \mathrm{~min}$ in a microwaver for antigen retrieval. After that, the sections were incubated with diluted rabbit polyclonal anti-Brf1 antibody (1:200 dilutions, Bethyl, USA) overnight at $4^{\circ} \mathrm{C}$ and a secondary antibody for $30 \mathrm{~min}$ at $37^{\circ} \mathrm{C}$. After the sections were incubated with DAB (3,3-diaminobenzidine) staining for $2 \mathrm{~min}$ for protein detection. The sections were counterstained with Mayer's hematoxylin to stain nucleus and were finally dehydrated and mounted. A negative control was obtained by replacing the primary antibody with a normal rabbit IgG.

\section{Evaluation of IHC}

The immunoreactivities were scored separately by two pathologists blinded to the clinical parameters using $\mathrm{H}$-score method. Tumor cell percentage were scored as $0 \sim 100 \%$ positive tumor cells. Staining intensity was categorized: 0 , no staining; 1 , weak staining; 2, moderate staining and 3, strong staining. The two individual parameters were multiplied. Then we can get an immunoreactivity score (IRS) ranging from 0 to $300 \%$. All results were confirmed by at least 2 pathologists in a double-blind analysis. An optimal cutoff value for high and low expression was determined by the median of IRS. All HCC patients were divided into two groups according to the median score of anti-BRF1 immunostaining

\section{Real time quantitative PCR (RT-qPCR) and transfection}

Liver cancer cell lines and non-tumor liver cell lines were grown to $85 \%$ confluence and starved in serum-free for 3-4 h. The cells were treated with $50 \mathrm{mM}$ ethanol for another $1 \mathrm{~h}$. Total RNAs of the cells and the liver tissues from human and mice were extracted with TRIzol reagent (Invitrogen). For siRNA transfection assays, AML-12 cells were cultured in 10\% FBS/DMEM-F12 medium as described previously [17]. Serum-free medium was added to each dish with Lipofectamine-2000 Brf1 siRNA or mmRNA complexes, and cells were further incubated for $4 \mathrm{~h}$ at $37^{\circ} \mathrm{C}$. The cells were incubated for $48 \mathrm{~h}$ before harvesting. Total RNA samples were quantified and reverse-transcribed. After first-strand cDNA synthesis, the real time qPCR (RT-qPCR) were performed with specific primers (Supplementary Table S4) and PCR reagent kits (Bio-Rad Biotech) in the ABI prism 7700 Sequence Detection System. Precursor of tRNA ${ }^{\mathrm{Leu}}$ and 5S rRNA transcripts and Brf1 mRNA were measured by RT-qPCR as described previously [17].

\section{Cell anchorage-independent growth}

$A M L-12$ cells $\left(1 \times 10^{4}\right.$ cells/well in 6-well plate $)$ were transfected with mismatch RNA (mmRNA) or Brf1 siRNA (Supplementary Table S3). The cells were suspended in $0.35 \%(\mathrm{w} / \mathrm{v})$ agar in $10 \% \mathrm{FBS} / \mathrm{DMEM} / \mathrm{F} 12$ with or without $50 \mathrm{mM}$ ethanol, $20 \mathrm{ng} / \mathrm{ml}$ EGF or both EGF and ethanol over a bottom layer of media with $0.5 \%$ (w/v) agar. Cells were fed fresh complete media with EGF or/and ethanol twice weekly. Colonies were counted 3-4 weeks or longer after plating as previously described [16].

\section{Immunoblot analysis}

Cells were treated with $50 \mathrm{mM}$ ethanol to extract total cell lysates. Protein concentrations of the resultant lysates were measured by the Bradford method using Fluostar Omega spectrometer (Cell Biology Core Laboratory of University of Southern California Research Center for Liver Diseases, P30 DK048522). Lysates (50 $\mu \mathrm{g}$ of protein) were subjected to immunoblot analysis as previously described [36-37]. Membranes were probed with specific antibodies as indicated. Hybond-P membrane was used for protein transfer. Bound primary antibody was visualized using horseradish peroxidase-conjugated secondary antibody (Vector Laboratories) and enhanced chemiluminescence reagents (Cell Signaling technology).

\section{Statistical analysis}

Statistical analysis was performed using SPSS software, version 17.0 (SPSS, Chicago, USA). The paired $\mathrm{T}$ test was used to analyze the significant difference of Brfl mRNA expression between HCC tissues and the 
paired adjacent normal tissues. Chi-square test was used to assess the association between Brfl expression and clinicopathological status of HCC patients. Survival curves for both Brf1 high-expression and Brf1 lowexpression patients were performed using the KaplanMeier analysis and the log-rank test for comparison. Univariate and multivariate regression analysis were performed with the Cox proportional hazards regression model to determine the effect of particular prognostic factors including Brfl expression on death events. A value of $\mathrm{P}<0.05$ was considered statistically significant.

\section{Abbreviations}

Brf1: TFIIB-related factor 1; HCC: hepatocellular carcinoma; Pol III genes: RNA polymerase III-dependent genes; IHC: immunohistochemistry; OS: overall survival;

\section{ACKNOWLEDGMENTS}

We want to thank Drs. M. R. Stallcup, and N. Kaplowitz (University of Southern California) for scientific discussion. This work was supported by National Institutes of Health grants AA017288, AA021114 and AA023247 to S.Z.

\section{CONFLICTS OF INTEREST}

The authors who have taken part in this study declared that they do not have anything to disclose regarding funding or conflict of interest with respect to this manuscript.

\section{REFERENCES}

1. Lieber, C. Hepatic, metabolic, and nutritional disorders of alcoholism: from pathogenesis to therapy. Crit Rev Clin Lab Sci. 2000; 37:551-584.

2. Seitz HK, Poschl G, Simanowski UA. Alcohol and cancer. Recent Dev Alcohol 1998;14:67-95.

3. Seitz HK, Stickel F. Molecular mechanisms of alcoholmediated carcinogenesis. Nat Rev Cancer 2007; 7:599-612.

4. Bagnardi V, Blangiardo M, La Vecchia C, Corrao G. A meta-analysis of alcohol drinking and cancer risk. $\mathrm{Br} J$ Cancer. 2001; 85:1700-1705.

5. Yuan JM, Govindarajan S, Arakawa K, Yu MC. Synergism of alcohol, diabetes, and viral hepatitis on the risk of hepatocellular carcinoma in blacks and whites in the U.S. Cancer 2004; 101:1009-1017.

6. Purohit V, Khalsa J, and Serrano J. Mechanisms of alcoholassociated cancers: introduction and summary of the symposium. Alcohol, 2005; 35:155-160.

7. McKillop IH, Schrum LW. Alcohol and liver cancer. Alcohol. 2005; 35:195-203. Review.
8. Lu S. and Mato J. Role of methionine adenosyltransferase and S-adenosylmethionine in alcohol-associated liver cancer Alcohol 2005; 35:227-234.

9. Borchert GM, Lanier W, Davidson BL. RNA polymerase III transcribes human microRNAs. Natl Struct Mol Biol. 2006; 13:1097-2001.

10. White, RJ. RNA polymerase III transcription and cancer. Oncogene 2004; 23: 3208-3216. Review.

11. Zhong S, Machida K, Tsukamoto H, Johnson DL. Alcohol induces RNA polymerase III-dependent transcription through c-jun by coregulating TBP and Brfl expression. $J$ Biol Chem. 2011; 286:2393-2401.

12. Machida K, Tsukamoto H, Mkrtchyan H, Duan L. Dynnyk A, Liu HM, Asahina K, Govindarajan S, Ray R, Ou JH, Seki E, Deshaies R, Miyake K, Lai MM. Toll-like receptor 4 mediates synergism between alcohol and HCV in hepatic oncogenesis involving stem cell marker Nanog. Proc Natl Acad Sci U S A. 2009; 106:1548-1553.

13. Zhong S, Johnson DL The JNKs differentially regulate RNA polymerase III transcription by coordinately modulating the expression of all TFIIIB subunits. Proc Natl Acad Sci U S A. 2009; 106: 12682-12687.

14. Johnson SA and Johnson DL. Enhanced RNA polymerase III-dependent transcription is required for oncogenic transformation. J Biol Chem. 2008; 283: 19184-19191.

15. Zhang Q, Jin J, Zhong Q, Yu XL, Levy D, Zhong S. ER $\alpha$ mediates alcohol-induced deregulation of Pol III genes in breast cancer cells. Carcinogenesis 2013; 34: 28-37.

16. Zhong Q, Shi G, Zhang Q, Zhang Y, Levy D, Zhong S. Role of phosphorylated histone $\mathrm{H} 3$ serine 10 in DENinduced deregulation of Pol III genes and cell proliferation and transformation. Carcinogenesis. 2013; 34: 2460-2469.

17. Zhang Q, Zhong Q, Evans AG, Levy D, Zhong S. Phosphorylation of histone $\mathrm{H} 3$ serine 28 modulates RNA polymerase III-dependent transcription. Oncogene 2011; 30:3943-3952.

18. Gao J, Aksoy BA, Dogrusoz U, Dresdner G, Gross B, Sumer SO, Sun Y, Jacobsen A, Sinha R, Larsson E, Cerami E, Sander C, Schultz N. Integrative analysis of complex cancer genomics and clinical profiles using the cBioPortal. Sci Signal, 2013; 6(269):pl1. doi: 10.1126/ scisignal.2004088.

19. Cerami E, Gao J, Dogrosoz U, Gross BE, Sumer SO, Aksoy BA, Jacobsen A, Byrne CJ, Heuer ML, Larson E, Antipin Y, Reva B, Goldberg AP, et al. The cBio cancer genomics portal: an open platform for exploring multidimensional cancer genomics data. Cancer Discov, 2012; 2:401-404.

20. Zhong Q, Shi G, Zhang Q, Lu L, Levy D, Zhong S. Tamoxifen represses alcohol-induced transcription of RNA polymerase III-dependent genes. Oncotarget 2014; 5: 12410-12417. doi: 10.18632/oncotarget.2678.

21. Kalra M, Mayee J, Assefa S, Kaul AK, Kaul R. Role of sex steroid receptors in pathobiology of hepatocellular carcinoma. World J. gastroenterol 2008; 14:5945-5961. 
22. Stice JP, Lee JS, Pechenino AS, Knowlton AA. Estrogen, aging and the cardiovascular system. Future Cardiol. 2009; 5: 93-103. Review.

23. Parkin DM, Bray F, Ferlay J, Pisani P. Global cancer statistics. CA Cancer J Clin. 2005; 55:74-108.

24. Naugler WE, Sakurai T, Kim S, Maeda S, Kim K, Elsharkawy AM, Karin M. Gender disparity in liver cancer due to sex different in MyD88-dependent IL-6 production. Science, 2007; 317:121-124.

25. Sarma DS, Rao RM, Rajalakshmi S. Liver tumor promotion by chemicals: models and mechanisms. Cancer Surv. 1986; 5:781-798.

26. Li Z, Tuteja G, Schug J, Kaestner KH. Foxa1 and Foxa2 are essential for sexual dimorphism in liver cancer Cell 2012; 148:72-83.

27. Zhong S, Zhang C, Johnson DL. Epidermal Growth Factor enhances cellular TBP levels and induces RNA polymerase I- and III-dependent gene activity. Mol. Cell. Biol., 2004; 24:5119-5129.

28. Johnson DL and Johnson SA. Cell biology, RNA metabolism and oncogenesis. Science 2008; 320:461-462.

29. Zhong Q, Shi G, Zhang Y, Lu L, Levy D, Zhong S. Alteration of BRCA1 expression affects alcohol-induced transcription of RNA Pol III-dependent genes. Gene. 2015; 556:74-79.

30. Woiwode A, Johnson SA, Zhong S, Zhang C. Roeder RG, Teichmann M, Johnson DL. PTEN represses RNA polymerase III-dependent transcription by targeting the TFIIIB complex. Mol Cell Biol. 2008; 28:4204-4214.

31. Winter AG, Sourvinos G, Allison SJ, Tosh K, Scott PH, Spandidos DA, White RJ. RNA polymerase III transcription factor TFIIIC2 is overexpressed in ovarian tumors. Proc Natl Acad Sci USA 2007; 97:12619-12624.

32. IARC. IARC Monographs on the Evaluation of Carcinogenic Risks to Humans, Vol 100, A Review of Human Carcinogens. Lyon, France: International Agency for Research on Cancer; 2011. http://monographs.iarc.fr/ ENG/Monographs/PDFs/index.php. Accessed November 2, 2011.

33. Cogliani VJ, Baan R, Straif K, Crosse Y, Lauby-Secretan B, El Ghissassi F, Bouvard V, Benbrahim-Tallaa L, Guha N, Freeman C, Galichet L, Wild CP. Preventable 33. exposures associated with human cancers. J Natl. Cancer Inst. 2011; 103:1827-1839.

34. Wang, S, Xu M, Li F, Wang X, Bower KA, Frank JA. Ethanol promotes mammary tumor growth and angiogenesis: the involvement of chemoattractant factor MCP-1. Breast Cancer Res Treat. 2012;133:1037-1048.

35. Wong, A.W. Dunlap SM, Holcomb VB, Nunez NP. Alcohol Promotes Mammary Tumor Development via the Estrogen Pathway in Estrogen Receptor Alpha-Negative HER2/neu Mice. Alcohol. Clin. Exp. Res, 2012, 36: 577-587.

36. Peng H, Zhu QS, Zhong S, Levy D. Transcription of the human microsomal epoxied hydrolase gene (EPHX1) is regulated by an HNF-4 $\alpha / \mathrm{CAR} / \mathrm{RXR} / \mathrm{PSA}$ complex. Biochim Biophys Acta 2013; 1829: 1000-1009.

37. Peng H, Zhu QS, Zhong S, Levy D. Transcription of the Human Microsomal Epoxide Hydrolase Gene (EPHX1) is Regulated by PARP-1 and Histone H1.2. Association with Sodium-Dependent Bile Acid Transport. PLOS ONE 2015; 10: e0125318. 